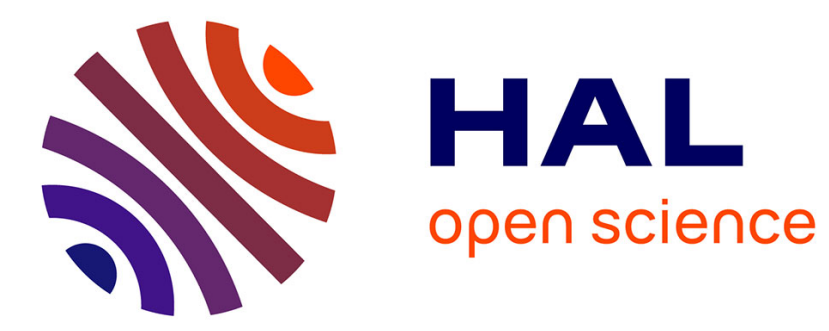

\title{
Control of interbank contagion under partial information
}

Hamed Amini, Andreea Minca, Agnès Sulem

\section{To cite this version:}

Hamed Amini, Andreea Minca, Agnès Sulem. Control of interbank contagion under partial information. SIAM Journal on Financial Mathematics, 2015, 6 (1), pp.24. hal-01027540v3

\section{HAL Id: hal-01027540 \\ https://inria.hal.science/hal-01027540v3}

Submitted on 21 Jan 2016

HAL is a multi-disciplinary open access archive for the deposit and dissemination of scientific research documents, whether they are published or not. The documents may come from teaching and research institutions in France or abroad, or from public or private research centers.
L'archive ouverte pluridisciplinaire HAL, est destinée au dépôt et à la diffusion de documents scientifiques de niveau recherche, publiés ou non, émanant des établissements d'enseignement et de recherche français ou étrangers, des laboratoires publics ou privés. 


\title{
Control of Interbank Contagion Under Partial Information*
}

\author{
Hamed Amini ${ }^{\dagger}$, Andreea Minca ${ }^{\ddagger}$, and Agnès Sulem ${ }^{\S}$
}

\begin{abstract}
We consider a stylized core-periphery financial network in which links lead to the creation of projects in the outside economy but make banks prone to contagion risk. The controller seeks to maximize, under budget constraints, the value of the financial system defined as the total number of projects. Under partial information on interbank links, revealed in conjunction with the spread of contagion, the optimal control problem is shown to become a Markov decision problem. We determine the optimal intervention policy by using dynamic programming. Our numerical results show that the value of the system depends on the connectivity in a nonmonotonous way: it first increases with connectivity and then decreases with connectivity. The maximum value attained depends critically on the budget of the controller. Moreover, we show that for highly connected systems, it is optimal to increase the rate of intervention in the peripheral banks rather than in core banks.
\end{abstract}

Key words. systemic risk, optimal control, financial networks

AMS subject classifications. 91B30, 91G50, 90B15, 90B50, 90B10, 91B15, 60J10

DOI. $10.1137 / 140981538$

1. Introduction. The financial system provides fundamental services to the outside economy. In the most simplified form, it channels capital from outside investors until it reaches real projects. While doing this, it has become intertwined globally. Today, we are far from the model with local banks whose retail depositors are funding local projects. In turn, the financial system is a global network of banks with a large number of peripheral banks and only a small number of core banks. These are the large banks that manage the global capital flows.

The recent crisis demonstrated that at the current levels of global interdependence of banks, systemic risks materialize. These are the risks that shocks in some part of the system are amplified by the system itself until they reach a global level and affect not only the stability of the financial system, but the outside economy as well.

Connectivity allows more credit to reach the periphery and real projects, while at the same time it creates systemic risk. In this paper we ask the following questions: How do these opposite effects compare at the level of the financial network, and how to define the value of the financial network to account for this dual aspect of connectivity? Can intervention by a lender of last resort achieve that the benefits of connectivity (projects) surpass the costs

\footnotetext{
${ }^{*}$ Received by the editors August 8, 2014; accepted for publication (in revised form) September 30, 2015; published electronically December 15, 2015. This work was partially funded by a research grant from the Europlace Institute of Finance.

http://www.siam.org/journals/sifin/6/98153.html

${ }^{\dagger}$ Swiss Finance Institute @ EPFL, Quartier UNIL-Dorigny, Extranef 249, 1015 Lausanne, Switzerland (hamed. amini@epfl.ch).

${ }^{\ddagger}$ School of Operations Research and Information Engineering, Cornell University, Ithaca, NY 14850 (acm299@ cornell.edu).

§INRIA Paris-Rocquencourt, Domaine de Voluceau, Rocquencourt, BP 105, Le Chesnay Cedex, 78153, France (agnes.sulem@inria.fr). 
associated with contagion? Should intervention be targeted towards core banks or towards peripheral banks?

To answer these questions we set up a stylized hierarchical model for a financial network with core banks and a set of peripheral banks. There is vast empirical evidence for a tiered structure of interbank networks for given countries; see, e.g., [9] for Austria, [11] for Brazil, [12] for Germany, etc. Core banks distinguish themselves from peripheral banks in that they are more interconnected and have access to an external creditor, e.g., money market funds. All banks have a certain number of projects that are funded by deposits, independently of the other banks. Moreover, peripheral banks have access to additional local projects but do not have access to the external creditor. The role of the network is to intermediate credit from the external creditor to the peripheral banks and fund these additional projects. We can consider the inaccessibility of the external creditor by the peripheral banks as a market friction.

We say that a bank fails when it no longer maintains credit to the other banks and no longer funds projects. This does not necessarily mean that the bank defaults; it may be that it hoards its liquidity as a precautionary measure against bank runs, as in [2]. As in [22, 21, 30], failures spread through the network from creditor to debtor banks: debtor banks who have seen a certain number of their credit lines withdrawn recall their credit lines.

Our model of failure cascade is a linear threshold model, interpreted as a model for liquidity hoarding. The threshold model for cascades in random graphs was introduced by [33] for social networks, and used in financial modeling for insolvency propagation (where insolvency means that liability exceeds assets) in [4], but also for liquidity hoarding modeling as in [22, 21]. A study by [30], who is the only one to our knowledge to consider at the same time both the liquidity hoarding cascade and the insolvency cascade on a real-world network, concludes that "both channels are relevant and that the credit line contagion channel is even more critical than the exposure channel."

In [2], the authors find that banks' willingness to provide credit to other banks is determined by their own rollover risk, which is the risk they will be unable to refinance. Rollover risk is in turn related to debt capacity. Reference [3] shows that debt capacity of banks' assets can fall far below fundamental value and it is not simply a function of the banks' creditworthiness. Indeed, [2] explains that during the crisis, even banks with the best credit quality find it difficult to borrow. It is beyond our scope to determine the debt capacity: in the case of a single bank with a continuum of creditors (the external creditor), the rollover decision and, consequently, the debt capacity are an outcome of a strategic game among creditors. It is driven by the firms' fundamentals but also by the beliefs on the probability of a run by the other creditors; see [25]. We take debt capacity exogenous and assume it already takes into account the fundamentals and the solvability of the banks.

We capture liquidity hoarding driven by debt capacity using the linear threshold model as follows. Each core bank is endowed with an initial distance to failure, which is its threshold. This is related to its debt capacity after an initial shock (that could be new information about the assets, about the solvency state of the bank, etc.), which essentially is a constraint on the number of credit lines it can raise from the outside creditor after the initial shock. Banks start with a certain number of credit lines from the outside creditor. With the failure propagation they may lose credit lines, and we consider that they can replace them up to a certain limit given by the debt capacity. So the distance to failure represents the remaining debt capacity. 
When the bank is at distance to failure zero, it has reached its debt capacity. Given that it can no longer raise liquidity, in absence of a lender of last resort, it is forced to withdraw credit. We, moreover, assume that at this point, it liquidates its projects.

Given its stylized nature, our model is not designed to be calibrated to a given network. Our goal is to bring insight into the relation between a regulator's control, the overall connectivity in the network, and the banks' position in the network, either at the periphery or at the core. It is beyond our scope to construct a theoretical model of liquidity hoarding, and we take as given that liquidity hoarding is a meaningful channel of contagion, as documented by studies conducted using central bank data; see, e.g., [30, 20]. ${ }^{1}$

We study the optimal intervention policy of a lender of last resort that injects liquidity into the system either in the core banks or in the peripheral banks after the initial shock. The lender of last resort would then optimally increase the debt capacity (and distance to failure) of the core banks, subject to a budget constraint. The criterion of the controller is the total value of the financial system at the end of the contagion, which is defined as the total number of projects of the banks. ${ }^{2}$

This problem is an optimization problem in high dimension. To make the problem tractable, the key idea is to formulate it as a Markov decision problem and to solve it by using dynamic programming. We achieve this by modeling the network as a random financial graph and revealing the lenders of failed banks one by one. The flow of information available to the controller is thus modeled as a link-revealing filtration. This filtration is monotonous in space: at each step, the failure cluster, i.e., the set of failed banks and their revealed links, increases and the controller learns of a new link to a failed bank. We learn the lenders of failed banks according to some arrival times. It is a realistic assumption that arrival times of the information about lenders of failed banks are distinct, and our model allows them to be arbitrarily close. At each of these arrival times, the link-revealing filtration increases. Given the observation of the failure cluster, the conditional law of the unobserved part of the network depends only on the current failure cluster and not its history.

Our main result (Theorem 3.3) states that the optimal control policy is Markovian in the observed failure cluster. Moreover, we prove a state space collapse result that states that the system can be described by a Markov chain with a lower dimension that gives the crosssectional distribution of the distance to failure. We show that the optimal control is feedback of the state of the lower-dimensional Markov chain. This provides us with a tractable framework for the optimal control problem, solvable by the dynamic programming approach.

In our numerical analysis, we vary the connectivity of the core banks, where zero connectivity means a financial system without any core banks that act as intermediaries; i.e., all projects are funded locally. We find that up to a certain level of connectivity, the value of the system increases with connectivity, and afterwards it decreases with connectivity. Our numerical results point to the existence of an "optimal" level of connectivity which depends on

\footnotetext{
${ }^{1}$ There are sophisticated game-theoretic models that explain the build-up of rollover risk, how bank runs (and in general decisions to withdraw funding) emerge out of collective optimal decisions of rational agents (see, e.g., [13, 25, 29, 2, 26, 28] in the case of one bank), while the extension to a network setting is an open question.

${ }^{2}$ Since we consider the aggregate value of the financial system, it is beyond our scope to consider the interest rate on the loans, which only has the effect of redistributing the value among banks.
} 
the initial number of failures in the system and the budget of the controller. At this optimal level of connectivity the system depends, in case there are initial failures, on the controller, and in particular on the availability of a strategy adapted to the flow of information generated by the spread of contagion. For highly connected systems with initial failures, even in the presence of intervention, the value of the system may fall below the value of the disconnected system.

We then analyze the intervention policy. In the low connectivity regime, the rate of intervention in core banks increases with connectivity. On the contrary, for highly connected systems, as we increase connectivity the optimal rate of intervention is higher for peripheral banks than for core banks.

We interpret these results as follows. In the low connectivity regime, there is little scope for contagion among core banks since nonfailed core banks can absorb the failure of a counterparty without any intervention. So the controller intervenes on the periphery, to stop contagion from the core to periphery. In the medium connectivity regime, intervention occurs on core banks: in this regime, the number of linkages among core banks increases, chains of intermediaries emerge, and contagion to nonfailed core banks is possible. In the high connectivity regime, there can be cycles in the subnetwork of core banks. For a fixed budget, there is a substantial risk that even if there is an intervention on core banks, contagion will spread due to these cycles among core banks and eventually reach all banks anyway. Therefore, it may be preferable in this case to inject in the peripheral banks directly and save them with certainty.

Our model can be used in several ways. We define the value of a system in the presence of a regulator and show that if connectivity is too large, then the value of the system drops even below that of the disconnected system. This is a surprising result, because more connectivity leads to more projects in general. So our results show that there are overconnected systems in which there is little scope for intervention and, under shock, all benefits from connectivity are erased by the costs associated to financial instability. The existence of this regime of overconnected systems whose value is even below that of disconnected systems is a new contribution. It means that if a system is in an overconnected regime, liquidity injections simply do not work, and in this regime the best policy is to reduce connectivity, possibly by splitting banks into smaller entities. Likewise, our results show that depending on the shock and the connectivity regime, the regulator would inject at a certain level of the tiered structure of the financial network, i.e., at either the periphery or the core.

All these have implications for moral hazard: Why not overconnect and get the benefits of connectivity if one would expect that the core banks always receive liquidity? The way banks connect would indeed change if it were given that in high connectivity regimes a regulator would have no effect, or if the level in the tiered structure where the regulator intervenes in various connectivity regimes would be given.

Network formation in the presence of a lender of last resort is one of the critical questions in finance. In recent work, [16] shows that core-periphery structures of financial networks appear endogenously in presence of bailouts. Creating the right incentives for the banks to reach desirable connectivities in the network remains an open question. Our model can be seen as a building block in this direction, since clearly the regulator's optimal actions are a critical ingredient of any incentive structure and drive the moral hazard issues related to network formation: When banks choose their position in the tiered structure of the network, 
their choice would take into account a regulator's optimal actions conditional on a shock. In sum, we propose a tool in the overall debate of how much connectivity should we have in presence of a lender of last resort, or even if there is scope for a lender of last resort.

Relation to previous literature. Our work is in the area of systemic risk; see [5, 19] and the references therein. This paper is related to a strand of works on various types of control of contagion in financial networks (see, e.g., [31]).

The random network model, based on the directed configuration model, was studied in the context of systemic risk in [4], who give asymptotic results on the scope of contagion in heterogenous networks, where core-periphery networks are a particular case of the heterogenous networks. Our focus here is not on the scope of contagion per se, but on the tradeoff between financial contagion, which is the price to pay for connectivity, and the creation of new projects, which is the benefit of connectivity. This tradeoff appears in the optimization criterion of the controller.

The idea of cost-benefit of the links is a key component in the financial network formation literature; see, e.g., [8] for a setting based on random graphs. Their focus is different, as they study the stability of a network formed by strategic banks that take into account the benefits and risks of connectivity.

The control of contagion in an interbank network is studied in [27] under full observation of the interbank network in a model that extends [15] to account for the interplay between insolvency and funding liquidity risk. The problem leads to a convex combinatorial optimization problem, which is tractable only if the controller intervenes only on the set of core banks. Contrary to this limitation, in the current model, under partial information revealed step by step, we prove a state space collapse theorem which allows for tractability.

In most of the previous works on contagion in directed financial networks, the sense of contagion is in the opposite sense of lending and the cascades are interpreted as cascades of insolvencies. In our paper, we consider similarly to the simulation studies $[22,21,30]$ that contagion goes in the same direction as the credit: the lines of credit are withdrawn when the lender fails.

Our work is also related to recent literature on spatial risk measures; see [17]. Similar to these works, the complexity of the model is spatial and not temporal, as our controller faces a problem in space as contagion may spread from the initially failed bank to the system, leading if no intervention to a larger number of failures.

Several previous works point to the link between connectivity and financial stability; e.g., $[6,4,1,23]$. Our work complements this literature by adding explicitly in the model the creation of projects driven by the connectivity of the core banks which act as intermediaries. A regulator weighs financial stability and the benefits of connectivity such as number of projects. For example, connectivity zero gives a stable network, but it also has little value. Prior works show that there is a nonmonotonous relation between connectivity and stability (or spread of contagion), but in these works the number of projects is fixed. In contrast, here we also integrate that more connected systems also have more projects. Our results are stronger, as we show that the effects of large connectivities (even if we create more projects) could lead to lower values of the system-lower than the value of the disconnected system even if a lender of last resort is present.

Within a reduced form approach to systemic risk, [24] proposes estimators for the hazard 
rate of a systemic event; $[18,10]$ model the evolution of the bank reserves using coupled diffusion processes: the liquidity provided by the central bank is captured by using a rate of borrowing and lending. The critical difference is that in our model banks do not only lend to each other but also fund projects. Consequently, while [10] finds that adding liquidity in the system does not affect systemic risk, in our model, the control affects the value of the financial network, which depends both on systemic risk and project creation.

\section{The model.}

2.1. General description. We fix a probability space under which the financial network is chosen among all networks in which core banks have a fixed connectivity and peripheral banks have connectivity at most one. Connectivity is a parameter and does not evolve in the time frame of the model, since we consider a short term illiquidity crisis. There is a numeraire represented by the value of one link, and normalized to 1 . If a peripheral bank connects to a core bank, the link funds its available project, also equal in value to one unit of the numeraire. The total number of links from the external creditor to the core banks is equal to the total number of links from the core banks to the peripheral banks.

While the connectivity is fixed, the counterparties (lenders and borrowers) of the core banks are randomly chosen. Note that it is sufficient to establish the counterparties of the core banks, since the peripheral banks can only connect to core banks, and the external creditor to core banks only.

Initially, banks are endowed with a certain resilience in front of the withdrawal of their credit, called distance to failure. Each time a credit line is withdrawn from a bank, its distance to failure decreases by 1 . When it reaches 0 , the bank fails and liquidates its projects and withdraws any credit from counterparties, so contagion occurs. We consider a worse case where the project is liquidated entirely and all credit lines are withdrawn, so we do not allow for partial liquidations or withdrawals. This is a tractability assumption. We call the failure cluster the set of failed banks and the credit lines that were withdrawn by them.

At each step, there is the observed failure cluster and an unobserved part of the financial network. Information arrival is conditioned on the spread of contagion: as long as there are failures in the system we learn the counterparties that have credit lines from the banks that are failed. When contagion stops, i.e., there are no more such links to be revealed, the system is no longer observed. As information arrives and a new link to a failed bank is revealed, the corresponding counterparty will decrease its distance to failure. When a link is revealed, in absence of intervention, the distance to failure of the counterparty decreases by 1 in case the bank was not failed and stays at 0 in case the counterparty already failed. In case there is an intervention, the banks remain at the same distance to failure. The controller can have a maximum number of interventions.

2.2. The system before the shock. We consider a financial system with $c$ core banks. We let $[c]:=\{1, \ldots, c\}$ be the set of core banks. There is a set of peripheral banks, formally represented by node $p$. There is also an external creditor, represented as node 0 . Each core bank holds $y_{c}$ in projects and each peripheral bank holds $y_{p}$ in projects, funded independently of all the other banks. In addition, there exists a financial network that intermediates credit lines from the external creditor to the peripheral banks. This is shown in Figure 1, in the 


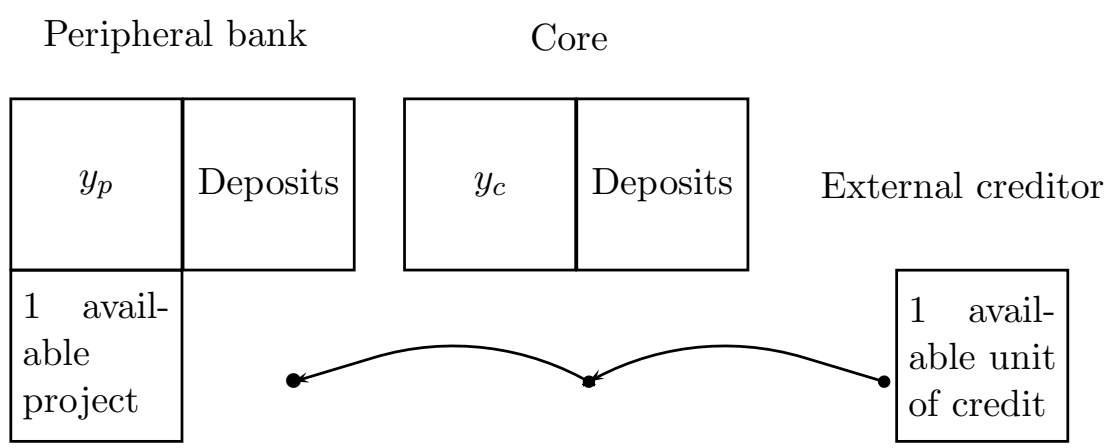

Figure 1. Credit intermediation by one core bank: one unit of credit from the external creditor is intermediated by the core bank and extended to a peripheral bank, which invests this unit of loan into one unit of additional project. In general, there may be multiple intermediary core banks.

particular case with one core bank and one peripheral bank. The core banks are thus distinguished from peripheral banks by their access to the external creditor. Accordingly, we need to interpret $y_{c}$ as an optimal level of direct funding chosen by a core bank: there is a cost associated to the search of projects, so after the number $y_{c}$ of projects funded directly they prefer to lend to other banks. It is beyond the scope of this paper to model the optimal choice of $y_{c}$ by the core banks.

Core banks have connectivity $\lambda$. They lend to $\lambda$ banks, which can be core or peripheral. In total there are

$$
m:=c \lambda
$$

credit lines extended by the core banks. An important ingredient in our model is the efficiency of the network, aimed at capturing that in general multiple core banks intermediate credit from the external creditor to the set of peripheral banks. We denote the network efficiency by $\pi$, with $\pi \in[0,1]$. Each core bank borrows from other core banks $(1-\pi) \lambda$ credit lines and $\pi \lambda$ credit lines from the external creditor. The set of peripheral banks borrows $m \pi$ credit lines from the core banks and there are $m(1-\pi)$ intercore links. As expected, in a network with $\pi=1$ (efficient intermediation), there are no intercore links and credit lines from the external creditor to the set of peripheral banks have only one intermediary.

In [4], it has been shown that the heterogeneity of the degrees (allowed to differ among the banks, both for the in-degrees and the out-degrees) plays an important role in the transmission of financial distress. Here, we consider that core banks have the same connectivity: our goal is not to analyze contagion in a given heterogenous network, but to understand how varying the connectivity of the core impacts the creation of projects, the contagion, and its control. Regularity is a common assumption in the literature that seeks insights on the role played by connectivity; see, e.g., [6, 1]. 
We represent the financial system as a directed unweighted network $([c] \cup\{0, p\}, \mathcal{E})$, where $\mathcal{E}$ represents the set of links. For a link $(i, j) \in \mathcal{E}, i$ is the lender and $j$ is the borrower. Links are unweighted; that is, there is a standardized value of a loan normalized to 1 and which plays in our model the role of a numeraire. We allow for multiple links between two banks. This corresponds to the case where there is more than one unit of a loan between the pair of banks.

The efficiency of the financial network is related to the number of new projects created by peripheral banks: Peripheral banks that receive one credit line from core banks fund one new unit of external project. The number of additional projects extended by the peripheral banks using the credit provided by core banks is given by

$$
m \pi=\#\{(i, p) \in \mathcal{E}, i \in[c]\} .
$$

We also have the following balance sheet equation, that the total number of links exiting the core banks is equal to the total number of links entering the core banks, i.e.,

$$
\#\{(i, j) \in \mathcal{E}, i \in[c], j \in[c] \cup\{p\}\}=\{(i, j) \in \mathcal{E}, i \in[c] \cup\{0\}, j \in[c]\},
$$

which in turn requires that

$$
m \pi=\#\{(0, j) \in \mathcal{E}, j \in[c]\},
$$

satisfied by construction since each core bank borrows $\lambda \pi$ credit lined from the external creditor.

In what follows, we will only consider the peripheral banks that are connected to the core banks, since the other ones are not subject to contagion or to the creation of new projects. There are $m \pi$ such peripheral banks, and each one holds one unit of project funded independently of the network and an additional project funded through the credit from core banks. In total, the number of units of project of the banks we consider, in absence of an initial shock, is given by

$$
\bar{J}:=c y_{c}+m \pi\left(y_{p}+1\right) .
$$

As connectivity increases in the network, the third term increases. However, as shown in Figure 2, contagion occurs in the same direction as the direction of the links, and therefore an increase of connectivity increases contagion risk. When a core bank fails, it withdraws its credit lines and liquidates its projects ( $y_{c}$ units). When a peripheral bank fails, it liquidates its projects ( $y_{p}$ units).

In what follows, we fix a probability space $\left(G_{c+2, m}, \mathbb{P}\right)$, where $G_{c+2, m}$ denotes the set of networks with $c+2$ nodes ( $c$ core banks, the external creditor, and the set of peripheral banks) and at most $m$ links. The financial network $\mathcal{E}$ and any partial observation of this network live on the space $G_{c+2, m}$.

Under the probability measure $\mathbb{P}$, the law of $\mathcal{E}$ is given as follows. Each core bank is assigned $(1-\pi) \lambda$ incoming half links (which corresponds to credit received from other core banks) and $\lambda$ outgoing half links (which corresponds to credit offered to core or peripheral banks). Node $p$ is assigned $\pi m$ incoming half links. In total we have $m=c \lambda$ outgoing half links and $c(1-\pi) \lambda+\pi m=c \lambda$ incoming half links. An outgoing half link represents an offer 


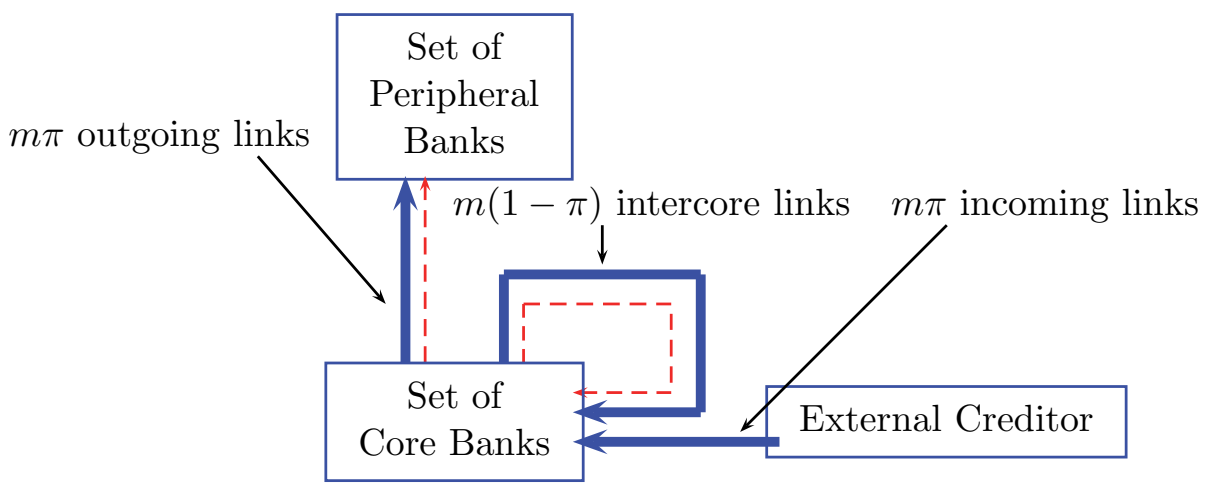

Figure 2. Direction of credit lines. More connectivity of the core banks amounts to more credit lines towards peripheral banks but also more cycles among core banks. Contagion happens in the direction of credit lines: when a bank fails it withdraws all credit lines from its borrowers.

of a credit line. An incoming half link represents a candidate to borrow one credit line. Only when these half links are matched, does this correspond to an effective loan.

The identity of these lenders and borrowers is random and results by a uniform matching of the $m$ outgoing half edges with the $m$ incoming half edges as in the directed configuration model; see [4] and the references therein. When an outgoing half link is matched with an incoming half link, a link is established.

This uniform draw establishes the borrowers of all core banks. Note that the network is not symmetric since not all core banks will have the same number of credit lines as other core banks, some of them will lend more to other core banks, and some of them will lend more to the set of peripheral banks. As such, the position of the core banks in the network differs, despite that they have the same connectivity.

Importantly, this draw also establishes all links among core banks. To ensure the balance equation (2), it is understood that each core bank is assigned $\pi \lambda$ credit lines from the external creditor. The outgoing links of the external creditor are not included in the matching, so as to ensure that peripheral banks do not have access to credit lines from the external creditor. In this paper, we use the well-known advantage of the configuration model; namely the configuration graph construction can be adapted to the cascade; see, e.g., [32].

Remark 1. Note that, given the observation of any subset of matched links, the unobserved matched links also have the same uniform distribution, and this conditional law depends on the observed set of matched links only through the number of matched links going out of core banks and not the history of the past matching. The subset of matched links can be in particular the cluster of defaults.

2.3. Dynamics of contagion and information. Each bank is endowed with a random variable denoted $\theta_{0}(i), i \in[n]$, which represents the number of credit lines that can be with- 
drawn from bank $i$, before bank $i$ fails. We call this variable "initial distance to failure" and distance 0 marks a failed bank.

We make the following assumption, which states that core banks are initially either failed or at the maximum distance to failure, which is consistent with the homogeneity across core banks. Peripheral banks have initially none of their credit lines withdrawn, so their initial distance to failure is 1 .

Assumption 1. The initial distance to failure of the core banks $\theta_{0}(i)$ takes values in $\left\{0, \vartheta_{\max }\right\}$ for all $i \in c$. The initial distance to failure of the peripheral banks is one.

We now endow the probability space $\left(G_{c+2, m}, \mathbb{P}\right)$ with a filtration $\left(\mathcal{G}_{k}\right)_{k \leq m}$ that models at the same time the financial contagion and the flow of information: at each step an outgoing link of a failed bank is revealed. This corresponds to a credit line that is withdrawn and consequently to a decrease in the distance to failure of the borrower at the end of the link. Rather than temporal, information has here a spatial dimension. We call this filtration the "link-revealing" filtration.

The failure cluster, started by the failure of a core bank, is given below by the set of failed core banks and the set of revealed links. It is defined as a random graph that evolves in the space $G_{c+2, m}$. At any point during the cascade dynamics, there are at most $c$ failed core banks and at most $m$ outgoing links of failed core banks.

Definition 2.1 (cascade of contagion and information). The cascade of failures and the flow of information is determined as follows:

\section{Initial condition.}

Let $\theta_{0}(i) \in\left\{0, \vartheta_{\max }\right\}, i \in[c]$, be given. Let the set of initially failed core banks be $F_{0}:=\left\{i \in[c] \mid \theta_{0}(i)=0\right\}$. Let the set of hidden links exiting initially failed banks be $\mathcal{H}_{0}:=\left\{(i, j) \in \mathcal{E}, i \in F_{0}\right\}$. Let the sigma-algebra representing the information available initially be $\mathcal{G}_{0}:=\sigma\left(\left(\theta_{0}(i), i \in[c]\right)\right)$.

\section{Dynamics.}

Let $k \in[1, m]$. If $\mathcal{H}_{k-1}=\emptyset$, Stop.

Otherwise: Let $\left(i_{k}, j_{k}\right) \in \mathcal{H}_{k-1}$ be a hidden link, chosen uniformly over the set $\mathcal{H}_{k-1} \cdot{ }^{3}$ Update the link-revealing filtration, $\mathcal{G}_{k}:=\mathcal{G}_{k-1} \vee \sigma\left(\left\{i_{k}, j_{k}\right\}\right)$. Set $\theta_{k}(i):=\theta_{k-1}(i) \forall i \in$ $[c] \backslash\left\{j_{k}\right\}$. If $j_{k} \in[c]$, set $\theta_{k}\left(j_{k}\right):=\theta_{k-1}\left(j_{k}\right)-1$.

(i) (No additional failure.) Set $F_{k}:=F_{k-1}$ and remove the revealed link from the set of hidden links

$$
\mathcal{H}_{k}:=\mathcal{H}_{k-1} \backslash\left\{\left(i_{k}, j_{k}\right)\right\}
$$

(ii) (Additional failure.) Set $F_{k}:=F_{k-1} \cup\left\{j_{k}\right\}^{4}$ and update the set of hidden links

$$
\mathcal{H}_{k}:=\mathcal{H}_{k-1} \backslash\left\{\left(i_{k}, j_{k}\right)\right\} \cup\left\{\left(j_{k}, j\right) \in \mathcal{E}\right\} .
$$

At any step of the cascade, the links exiting failed banks are partitioned into two categories: revealed and hidden. For tractability reasons, we need that only one of these links is revealed

\footnotetext{
${ }^{3}$ Note that when the hidden link $\left(i_{k}, j_{k}\right)$ is revealed at time $k$, all links exiting the failed bank $i_{k}$, i.e., $\left\{\left(i_{k}, j\right) \in \mathcal{E}\right\}$, are either revealed or in the set of hidden links. If they are hidden, they will remain in the set of hidden links, until revealed one by one.

${ }^{4}$ Note that at any step $k \in[0, m]$, we have $F_{k}=\left\{i, \theta_{k}(i)=0\right\}$.
} 
at one time. Therefore, we choose them randomly one by one. During the cascade, new information arrives in the system when a new hidden link is revealed. Since the new revealed link $\left(i_{k}, j_{k}\right)$ is exiting a failed node $i_{k}$, the revealed counterparty $j_{k}$ (if not failed already) will have a credit line withdrawn and its distance to failure reduced by one. Each link revealed is removed from the set of hidden links (see (5) and (4)). If the counterparty fails, i.e., its distance to failure reaches zero, then the set of failures increases. If this failed bank is a peripheral bank, it does not have any additional links. If it is a core bank, all its links are not revealed immediately, but they become hidden links. Thus the number of hidden links jumps up (see (5)).

In sum, the number of hidden links decreases by one at each step of the algorithm but jumps up when a core bank fails. Note also that the stopping time $T$ at which all hidden links are revealed is always smaller than $m=c \lambda$, the total number of outgoing links of core banks.

The set of hidden links $\mathcal{H}_{k}$ is not adapted to the filtration generated by the revealed links $\mathcal{G}_{k}$. This is what justifies the notion of "partial information" in our model. Each hidden link $\left(j_{k}, j\right)$, added to the set of hidden links in $(5)$, represents a lending relation between a failed node $j_{k}$ and one of its debtors. This link will belong to failure cluster, but is not revealed at step $k$ when $j_{k}$ fails. It will be revealed later. In this sense, the distance to failure $\theta_{k}(i)$ is only an observed distance to failure, since the hidden link will decrease the distance to failure of $i$ later. Likewise, $F_{k}$ is only an observed set of failures.

3. Optimal intervention problem. We now consider the previous setting, but we assume that there exists a controller that intervenes a maximum number of times $M$ (which represents the budget constraint) at steps $1 \leq k \leq m$. When the controller intervenes on a bank, its distance to failure increases by 1 . We interpret the controller as a lender of last resort: in absence of intervention a bank will see its credit line withdrawn by a failed bank. With intervention, this credit will be replaced by a line of credit from the lender of last resort.

3.1. Contagion and information under intervention. We consider the case when the controller may intervene at each step only on one bank, which we understand is the end of the revealed link. For this type of control, we introduce the control set

$$
\mathcal{U}_{M}:=\left\{\left(u_{k} \in\{0,1\}, 1 \leq k \leq m\right), \sum_{1 \leq k \leq m} u_{k} \leq M\right\} .
$$

We will show later (Proposition 3.4) that for the optimization criterion that we consider, this space is sufficient; i.e., it would not be optimal for the controller to intervene on multiple banks at the same time or on banks which are not the end of the revealed link.

We now define the controlled cascade of information and contagion, where the control process takes values in $\mathcal{U}_{M}$ and is adapted to the link-revealing filtration.

Definition 3.1 (controlled cascade of information and contagion). The controlled cascade of information and contagion is determined as follows:

\section{Initial condition.}

Let $\theta_{0}^{u}(i) \in\left\{0, \vartheta_{\max }\right\}, i \in[c]$, be given. Let the set of initially failed banks be $F_{0}^{u}:=$ $\left\{i \in[c] \mid \theta_{0}(i)^{u}=0\right\}$. Let the set of hidden links exiting initially failed banks be $\mathcal{H}_{0}^{u}:=\left\{(i, j) \in \mathcal{E}, i \in F_{0}^{u}\right\}$. Let the sigma-algebra representing the information available initially be $\mathcal{G}_{0}^{u}:=\sigma\left(\left(\theta_{0}^{u}(i), i \in[c]\right)\right)$. Set $v_{0}^{u}(i):=0, i \in[c] \cup\{p\}$. 


\section{Dynamics.}

Let $k \in[1, m]$. If $\mathcal{H}_{k-1}^{u}=\emptyset$, Stop.

Otherwise: Let $\left(i_{k}^{u}, j_{k}^{u}\right) \in \mathcal{H}_{k-1}^{u}$ be a hidden link chosen uniformly over the set $\mathcal{H}_{k-1}^{u}$. Update the link-revealing filtration, $\mathcal{G}_{k}^{u}=\mathcal{G}_{k-1}^{u} \vee \sigma\left(\left\{i_{k}^{u}, j_{k}^{u}\right\}\right)$. Set $\theta_{k}^{u}(i):=\theta_{k-1}^{u}(i) \forall i \in$ $[c] \backslash\left\{j_{k}^{u}\right\}$.

Choose $u_{k} \mathcal{G}_{k}^{u}$-measurable, with values in $\{0,1\}$, such that $\sum_{i \in[c] \cup\{p\}} v_{k}^{u}(i) \leq M$, where

$$
v_{k}^{u}(i):=v_{k-1}^{u}(i)+u_{k} \mathbb{1}_{i=j_{k}^{u}}, i \in[c] \cup\{p\} .
$$

$\operatorname{Set} \theta_{k}^{u}\left(j_{k}^{u}\right):=\theta_{k-1}^{u}\left(j_{k}^{u}\right)-1+u_{k}$.

(i) (Additional failure.) If $\theta_{k}^{u}\left(j_{k}^{u}\right)=0$ and $\theta_{k-1}^{u}\left(j_{k}^{u}\right)=1$, then $F_{k}^{u}:=F_{k-1}^{u} \cup\left\{j_{k}^{u}\right\} ; \mathcal{H}_{k}^{u}:=\mathcal{H}_{k-1}^{u} \backslash\left\{\left(i_{k}^{u}, j_{k}^{u}\right)\right\} \cup\left\{\left(j_{k}^{u}, j\right) \in \mathcal{E}\right\}$.

(ii) (No additional failure.) Otherwise $F_{k}^{u}:=F_{k-1}^{u} ; \mathcal{H}_{k}^{u}:=\mathcal{H}_{k-1}^{u} \backslash\left\{\left(i_{k}^{u}, j_{k}^{u}\right)\right\}$.

Note that indeed the filtration defined above is the link-revealing filtration:

$$
\mathcal{G}_{k}^{u}=\sigma\left(\left\{\left(i_{l}^{u}, j_{l}^{u}\right), 0 \leq l \leq k\right\},\left(\theta_{0}(i)\right)_{i \in[n]}\right) .
$$

It is immediate to see from the definition above that $\left(\theta_{k}^{u}(i)\right)_{i \in[n]}$ is $\mathcal{G}_{k}^{u}$-measurable.

Based on the values of $\left(v_{k}^{u}(i), i \in[c]\right),\left(\theta_{k}^{u}(i), i \in[c]\right)$ we can determine at any time $k<T^{u}$ how many credit lines were withdrawn up to time $k$. Since at each step $1, \ldots, k$ a credit line was withdrawn from either a core or a peripheral bank, there are in total $k$ credit lines withdrawn. For core banks, each lost credit line leads to a decrease of the distance to failure, unless there was intervention. In total, we can compute that the core bank $i$ lost a number of $\theta_{0}^{u}(i)+v_{k}^{u}(i)-\theta_{k}^{u}(i)$ of its credit lines from the core banks up to time $k$. The number of credit lines withdrawn from peripheral banks by failed core banks up to time $k$ is thus given by

$$
k-\sum_{i \in[c]}\left(\theta_{0}^{u}(i)+v_{k}^{u}(i)-\theta_{k}^{u}(i)\right)
$$

so we can define the number of remaining credit lines from core to peripheral up to time $k$ :

$$
P_{k}^{u}:=m \pi-\left(k-\sum_{i \in[c]}\left(\theta_{0}^{u}(i)+v_{k}^{u}(i)-\theta_{k}^{u}(i)\right)\right) .
$$

We have the following lemma.

Lemma 3.2. Let $k<T_{k}^{u}$. The node $j_{k+1}^{u}$, representing the end node of the link $\left(i_{k+1}^{u}, j_{k+1}^{u}\right)$ chosen uniformly over the set $\mathcal{H}_{k}^{u} \neq \emptyset$, has the following law conditional on the sigma-algebra $\mathcal{G}_{k}^{u}:$

$$
\mathbb{P}\left[j_{k+1}^{u}=i \mid \mathcal{G}_{k}^{u}\right]= \begin{cases}\frac{\left((1-\pi) \lambda-\left(\theta_{0}^{u}(i)+v_{k}^{u}(i)-\theta_{k}^{u}(i)\right)\right.}{m-k}, & i \in[c], \\ \frac{P_{k}^{u}}{m-k}, & i=\{p\} .\end{cases}
$$

Proof. Note that $\mathcal{H}_{k}^{u} \neq \emptyset$ for $k<T^{u}$. At step $k$ one credit line is withdrawn uniformly over the set of links that were not revealed and whose start node is failed. Using Remark 1 for our random graph model, the conditional law of the identity of the end node (determined 
by the chosen end half link) is given by the uniform matching to the remaining half links. We check that the probabilities above correspond to the number of the remaining half links. For core banks, the numerator represents the number of available incoming half links of a node, given by the difference between the initial $(1-\pi) \lambda$ credit lines from other core banks and the withdrawn $\theta_{0}^{u}(i)+v_{k}^{u}(i)-\theta_{k}^{u}(i)$ credit lines.

Similarly, for the peripheral banks, the conditional law depends on the number of credit lines that were withdrawn. By definition of (6), the sum of these conditional probabilities is one.

It is immediate from the definition of the cascade that given $\left(v_{k}^{u}(i), i \in[c] \cup\{p\}\right),\left(\theta_{k}^{u}(i)\right.$, $i \in[c]$ ), the "new observation" $j_{k+1}^{u}$ and the action $u_{k+1}$ taken at step $k+1$, we can determine $\left(v_{k+1}^{u}(i), i \in[c] \cup\{p\}\right),\left(\theta_{k+1}^{u}(i), i \in[c]\right)$. Given the previous lemma, and that the control is adapted to the link revealing filtration, which is the same as the history of the process $\left(v_{k}^{u}(i), i \in[c] \cup\{p\}\right),\left(\theta_{k}^{u}(i), i \in[c]\right)$, the system is Markovian in the current value of the process $\left(\left(v_{l}^{u}(i), i \in[c] \cup\{p\}\right),\left(\theta_{l}^{u}(i), i \in[c]\right)\right)_{0<l<k}$ if the optimization criterion can be written only using the current value of this process. This is the case we study below, so from now on we refer to $\left(v_{k}^{u}(i), i \in[c] \cup\{p\}\right),\left(\theta_{k}^{u}(i), i \in[c]\right)$ as the state of the system at time $k$.

3.2. Optimal control problem. We consider as optimization criterion the value of the financial system at the end of the cascade process. This is defined similarly to the case without an initial shock in (3),

$$
J_{T^{u}}^{u}=\#\left\{i \in[c], \theta_{T^{u}}^{u}(i)>0\right\} y_{c}+\left(\#\left\{(i, p), i \in[c], \theta_{T^{u}}^{u}(i)>0\right\}+v_{T^{u}}^{u}(p)\right)\left(y_{p}+1\right) .
$$

The quantity $\left(\#\left\{(i, p), i \in[c], \theta_{T^{u}}^{u}(i)>0\right\}+v_{T^{u}}^{u}(p)\right)$ represents the number of peripheral banks that keep their funding (either because the line was not withdrawn or because of intervention), so the last term represents the total value of the projects of these peripheral banks. Note that in the first term of (8), we count only the projects of nonfailed core banks, since we considered that the projects of failed banks are liquidated. ${ }^{5}$

We can define now the optimal control problem.

Problem 1 (optimal control under partial information). We have

$$
\left.\Phi_{0}:=\max _{u \in \mathcal{U}^{a}} \mathbb{E}\left(J_{T^{u}}^{u}\right) \mid \mathcal{G}_{0}\right),
$$

where $\mathcal{U}^{a}$ denotes the set of $\left(\mathcal{G}_{k}^{u}\right)_{1 \leq k \leq m}$-adapted processes with values in $\mathcal{U}_{M}$.

Remark 2. Note that, in general, the solution to the optimal control problem defined above depends on the order in which the hidden links are revealed. We can indeed interpret this ordering in the following continuous-time model in which events are spread over time: After a bank fails, the withdrawal of the credit lines from its debtors is not instantaneous, but there is a time span between a bank's failure and the time when a debtor bank will have its credit line withdrawn. In particular, for any bank $j$, let $T_{j}(i)$ denote the time span between $i$ 's failure

\footnotetext{
${ }^{5}$ Maximizing the value of the financial system is equivalent to minimizing the dead-weight losses associated to the liquidation of the projects. In aggregate, these losses are borne by the banks' shareholders and the external creditor. Moreover, our definition of the value of the financial system is in the case of zero recovery rates for the external project. We could introduce a recovery rate, but this would not change our results which are essentially qualitative.
} 
and the time when $j$ has its credit line from $i$ withdrawn (i.e., the time needed to reveal the hidden link from $i$ to $j$ ). All initial failures, as defined in the initial condition of the cascade in Definition 3.1, occur at time 0.

Considering that $\left\{T_{j}(i)\right\}_{0 \leq i, j \leq n}$ are independent and identically distributed (i.i.d.) exponentially distributed random variables, the $k$ th credit line that is withdrawn (corresponding to the $k$ th event of the continuous time contagion process) has the same law as the $k$ th revealed link, i.e., $\left(i_{k}, j_{k}\right)$ in Definition 3.1. By the memoryless property of the exponential distribution, the transition probabilities in Lemma 3.2 remain the same. Step $k$ of the cascade in Definition 3.1 corresponds thus to event $k$ of the continuous time cascade process and the state of the system at step $k$ corresponds to the state of the system (in continuous time), after the $k$ th event. Since our optimization criterion does not depend on time, but only on the state of the system at the end of the cascade, it is sufficient for us to work with the embedded discrete time Markov chain.

This continuous time model is essentially a search model. At each event time, a counterparty of a failed bank is chosen randomly (as the end node of a hidden link, chosen uniformly over the set of hidden links). The arrival of events corresponds to a Poisson process with state-dependent intensity, a common setup in the search models; see, e.g., [14].

3.3. State space collapse theorem. Since the control space $\mathcal{U}^{a}$ is finite, there exists an optimal control. However, a priori the state of the system has a high dimension, which would make the optimization problem intractable. In this section, we prove the main result of the paper, namely a state space collapse theorem: contagion and the optimal control can be determined using some aggregates of the state of the system. Moreover, we show that these aggregates represent a controlled Markov chain.

Recall that the position of the core banks is different and only partially observed. For nonfailed banks, what determines their systemic risk is their current distance to failure, since this is determined by the number of credit lines that were withdrawn. Nonfailed core banks at the same distance to failure have the same risk and are similar from the point of view of the controller, who cannot observe their borrowers.

Therefore, we need only keep track of their number during the cascade, rather than their individual state, which is a significant reduction in the dimension of the state of the system. Since we assumed that all core banks have the same connectivity, we need only keep track of the number of core banks at each distance to failure.

Starting from the state variables

$$
\left(\left(v_{k}^{u}(i), i \in[c] \cup\{p\}\right),\left(\theta_{k}^{u}(i), i \in[c] \cup\{p\}\right)\right),
$$

we define the following aggregates:

$$
A_{k}^{u}:=\left(\left(C_{k}^{u}(\vartheta), \vartheta=1, \ldots, \vartheta_{\max }\right), P_{k}^{u},\left(I_{k}^{c, u}(\vartheta), \vartheta=1, \ldots, \vartheta_{\max }\right), I_{k}^{p, u}\right), \quad k=0, \ldots, m
$$

with

- $C_{k}^{u}(\vartheta), \vartheta=1, \ldots, \vartheta_{\max }$ : the number of core banks having distance to failure equal to $\vartheta$ at step $k$ :

$$
C_{k}^{u}(\vartheta):=\#\left\{i \in[c], \theta_{k}^{u}(i)=\vartheta\right\}
$$


- $P_{k}^{u}$ : the number of credit lines from core to peripheral which are not withdrawn, introduced in (6);

- $I_{k}^{c, u}(\vartheta):=\sum_{i \in[c], \theta_{k}(i)=\vartheta} v_{k}^{u}(i)$ : the number of interventions up to step $k$ on core banks having distance to failure equal to $\vartheta$.

- $I_{k}^{p, u}=v_{k}^{u}(p)$ : the number of interventions up to step $k$ on peripheral banks.

For all $k=1, \ldots, m, A_{k}^{u}$ takes values in

$$
\mathcal{A}:=\left\{\left(x_{c}, x_{p}, i_{c}, i_{p}\right) \in[0, c]^{\vartheta_{\max }} \times[0, m \pi] \times[0, M]^{\vartheta_{\max }+1} \mid \sum_{\vartheta=1}^{\vartheta_{\max }} x_{c}(\vartheta) \leq c, \sum_{\vartheta=1}^{\vartheta_{\max }} i_{c}(\vartheta)+i_{p} \leq M\right\} .
$$

In what follows, we will use the following aggregate of the new observation and will refer to this also as "the new observation" at time $k$ :

$$
Y_{k}:=\left(\mathbb{1}_{j_{k}^{u} \in[c]}, \theta_{k}\left(j_{k}^{u}\right)^{+} \mathbb{1}_{j_{k}^{u} \in[c]}\right)
$$

with values in

$$
\mathcal{O}:=\left\{(1, \vartheta), 1 \leq \vartheta \leq \vartheta_{\max }\right\} \cup\{(1,0),(0,0)\},
$$

$(1, \vartheta), \vartheta>1$, is the case when we choose a nonfailed core bank at distance to failure $\vartheta ;(1,0)$ is the case when we choose a failed core bank; and $(0,0)$ is the case when we choose a peripheral bank, in total $\vartheta_{\max }+2$ possible values. We have the following main theorem.

Theorem 3.3 (state space collapse). Let $\mathcal{U}^{\text {Feedback }}$ be the set of feedback controls $u=\left(u_{k}\right)_{k \in[1, m]}$ $\in \mathcal{U}^{a}$ of the form

$$
u_{k}=U_{k}\left(A_{k-1}^{u}, Y_{k}\right) \text { for } 1 \leq k \leq m,
$$

with $U_{k}$ a deterministic function.

For any feedback control $u \in \mathcal{U}^{\text {Feedback }}, A^{u}$ is a Markov chain, with respect to its own filtration $\left(\sigma\left(\left(A_{l}^{u}\right)_{l \leq k}\right)\right)_{k \leq m}$. Moreover, there exists an optimal control $u^{*} \in \mathcal{U}^{a}$ for Problem 1 and we have that $u^{*} \in \mathcal{U}^{\text {Feedback}}$.

Proof. The proof of the theorem necessitates several steps. We show the following for any adapted control $u \in \mathcal{U}^{a}$.

(i) The criterion $J_{T^{u}}^{u}$ can be written using only the aggregate variables at the stopping time $T^{u}, A_{T^{u}}^{u}$ :

First, note that the stopping time representing the end of the cascade $T^{u}$ can be written, using the aggregate state, as the following exit time:

$$
T^{u}=\inf \left\{k \mid\left(c-\sum_{\vartheta=1}^{\vartheta_{\max }} C_{k}^{u}(\vartheta)\right) \lambda-k=0\right\} .
$$

This represents the time in which there are no more hidden links in Definition 3.1 of the cascading failures since the number of hidden links that exit failed core banks at step $k$ is given by $\# \mathcal{H}_{k}=\left(c-\sum_{\vartheta=1}^{\vartheta_{\max }} C_{k}^{u}(\vartheta)\right) \lambda-k$, i.e., the total number of links outgoing from failed core banks minus the number of revealed links $k$. Therefore, we can write

$$
\mathbb{1}_{k<T^{u}}=\mathbb{1}_{\left(c-\sum_{\vartheta=1}^{\vartheta \max } C_{k}^{u}(\vartheta)\right) \lambda-k>0} .
$$


Second, the criterion in (8) can be written in terms of the aggregate state. The criterion

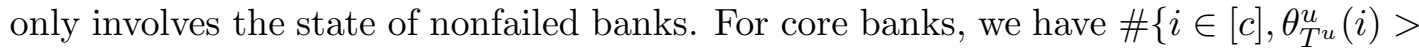
$0\}=\sum_{\vartheta=1}^{\vartheta_{\max }} C_{T^{u}}^{u}(\vartheta)$. Moreover, the number of remaining links from core to peripheral banks by the end of the cascade writes as $P_{T^{u}}^{u}=\#\left\{(i, p) \in \mathcal{E}, i \in[c], \theta_{T^{u}}^{u}(i)>0\right\}$, since by the end of the cascade all the links in the set $\left\{(i, p) \in \mathcal{E}, i \in[c], \theta_{T^{u}}^{u}(i) \leq 0\right\}$ are revealed. We have

$$
\begin{aligned}
J_{T^{u}}^{u} & :=\sum_{\vartheta=1}^{\vartheta_{\max }} C_{T^{u}}^{u}(\vartheta) y_{c}+\left(P_{T^{u}}^{u}+I_{T^{u}}^{p, u}\right)\left(y_{p}+1\right) \\
& =J\left(A_{T^{u}}^{u}\right),
\end{aligned}
$$

with

$$
J(x):=\sum_{\vartheta=1}^{\vartheta_{\max }} x_{c}(\vartheta) y_{c}+\left(x_{p}+i_{p}\right)\left(y_{p}+1\right), \quad x=\left(x_{c}, x_{p}, i_{c}, i_{p}\right) \in \mathcal{A} .
$$

(ii) The transition probabilities of the aggregate variables $A_{k+1}^{u}$ depend only on the previous state $A_{k}^{u}$, the new (aggregate) observation $Y_{k+1}$, and the control at step $k+1$, $u_{k+1}$.

For $k \in\left[T^{u}, m\right]$,

$$
A_{k+1}^{u}=A_{k}^{u}
$$

For $k \in\left[0, T^{u}\right)$, we have the following transition probabilities for the aggregate state $A_{k}^{u}$, where we only specify the changes in the aggregate state variables. Note, in particular, that if $Y_{k+1}=(1,0)$, i.e., we chose a failed core bank, then the aggregate state does not change.

If $u_{k+1}=0$,

$$
\begin{cases}C_{k+1}^{u}(\vartheta)=C_{k}^{u}(\vartheta)-1, C_{k+1}^{u}(\vartheta-1)=C_{k}^{u}(\vartheta-1)+1 & \text { if } Y_{k+1}=(1, \vartheta), \vartheta>1 \\ C_{k+1}^{u}(1)=C_{k}^{u}(1)-1 & \text { if } Y_{k+1}=(1,1), \\ P_{k+1}^{u}=P_{k}^{u}-1 & \text { if } Y_{k+1}=(0,0) .\end{cases}
$$

The second line above represents the case when there is a new failure.

If $u_{k+1}=1$,

$$
\begin{cases}I_{k+1}^{c, u}(\vartheta)=I_{k}^{c, u}(\vartheta)+1 & \text { if } Y_{k+1}=(1, \vartheta), \vartheta \geq 1, \\ P_{k+1}^{u}=P_{k}^{u}-1 ; I_{k+1}^{p, u}=I_{k}^{p, u}+1 & \text { if } Y_{k+1}=(0,0) .\end{cases}
$$

(iii) The conditional law of the new observation $Y_{k+1}=\left(\mathbb{1}_{j_{k}^{u} \in[c]}, \theta_{k}\left(j_{k}^{u}\right)^{+} \mathbb{1}_{j_{k}^{u} \in[c]}\right)$ on the sigma-algebra $\mathcal{G}_{k}^{u}$ depends only on the aggregate variable $A_{k}^{u}$.

Indeed, by summing the transition probabilities in Lemma 3.2 over all core banks at 
distance to failure $\vartheta \in\left[1, \vartheta_{\max }\right]$ we obtain

$$
\begin{aligned}
p_{k}(1, \vartheta):=\mathbb{P}\left[Y_{k+1}=(1, \vartheta) \mid \mathcal{G}_{k}^{u}\right]=\frac{C_{k}^{u}(\vartheta)\left((1-\pi) \lambda-\left(\vartheta_{\max }-\vartheta\right)-I_{k}^{c, u}(\vartheta)\right)}{m-k} \\
\forall \vartheta=1, \ldots, \vartheta_{\max }, \\
p_{k}(0,0):=\mathbb{P}\left[Y_{k+1}=(0,0) \mid \mathcal{G}_{k}^{u}\right]=\frac{P_{k}^{u}}{m-k},
\end{aligned}
$$

where in the first equation we used Assumption 1 and the observation that for any bank with distance to failure $\vartheta>0$ at time $k$ the initial distance to failure was necessarily $\vartheta_{\max }$. The probability that the new observation corresponds to a failed core bank is $1-\sum_{\vartheta=1}^{\vartheta_{\max }} p_{k}(1, \vartheta)-$ $p_{k}(0,0)$ and in this case the system remains in the same state.

It is now clear from (12) and points (ii) and (iii) above that we can write

$$
A_{k+1}^{u}=f\left(A_{k}^{u}, Y_{k+1}, u_{k+1}\right),
$$

with the $\mathcal{G}_{k}^{u}$ - conditional law of the new observation $Y_{k+1}$ depending only on $A_{k}^{u}$ and $u$ an $\left(\mathcal{G}_{k}^{u}\right)$ - adapted control. It follows from (15) that $A_{k}^{u}$ is a Markov chain (adapted to its own filtration) if moreover $u \in \mathcal{U}^{\text {Feedback }}$. By the standard theory of stochastic control in discrete time (see, e.g., [7]), the last statement of the previous theorem follows from (15) and point (ii) above.

The following proposition allows us to further reduce the dimension of the problem, under criterion (8). Regarding the interventions on the core banks, we need only keep track of their total number. It will be understood that interventions on core banks always occur at distance to failure 1 and there are no interventions on core banks at distance $\vartheta>1$ :

$$
I_{k}^{c, u}(\vartheta)=0, \quad k=0, \ldots, m, \quad \vartheta>1 .
$$

This will not necessarily be the case if we consider other optimality criteria. The following proposition also states that we lose nothing by restricting the control to the set $\mathcal{U}_{M}$ rather than the larger control set $\overline{\mathcal{U}}_{M}$ which allows intervention on multiple banks at the same time defined as

$$
\overline{\mathcal{U}}_{M}:=\left\{\left(u_{k} \in\{0,1\}^{c+1}, 1 \leq k \leq m\right), \sum_{1 \leq k \leq m} \sum_{i \in[c] \cup\{p\}} u_{k}(i) \leq M\right\} \text { with } \mathcal{U}_{M} \subseteq \overline{\mathcal{U}}_{M} .
$$

Proposition 3.4. Consider the optimization criterion $J_{T^{u}}^{u}$ given by (8) and the Problem 1 with the control set $\overline{\mathcal{U}}_{M}$. Then, for $1 \leq k \leq m, u_{k}(i)=0$ if $i \neq j_{k}^{u}$ or if $i=j_{k}^{u}$ and $\theta\left(j_{k}^{u}\right)>1$.

Proof. The dependence of the criterion $J_{T^{u}}^{u}$ on the set of core banks $\left\{i \in[c] \mid \theta_{T^{u}}(i)>0\right\}$ is only through its cardinal. Any given core bank will modify the state of the other banks only after the time it fails. (The set of hidden links $\mathcal{H}_{k}$ that drives the contagion grows only when there is a new failure of a core bank.) Moreover, each core bank that fails is chosen randomly from the set of core banks at distance to failure 1. Before this time, each of these banks' distance to failure decreased by 1 each time it was chosen as an end node of a hidden link. Let $\tau(i):=\inf \left\{k, j_{k}^{u}=i, \theta_{k}^{u}(i)=1\right\}$, possibly $\infty$. Then for $k<\tau(i), u_{k}(i)=0$. 


\section{Numerical analysis.}

4.1. Dynamic programming method. Using the state space collapse Theorem 3.3 and Proposition 3.4, the problem is now tractable and can be solved using dynamic programming. We have the following result.

Proposition 4.1. The value function for Problem 1 at step 0 is given by $\Phi_{0}=\phi_{0}\left(A_{0}\right)$ with $\phi_{k}$ defined backward recursively by the Bellman equation for all $x=\left(x_{c}, x_{p}, i_{c}, i_{p}\right) \in \mathcal{A}$ and $k=m, \ldots, 0$,

$$
\phi_{k}(x)= \begin{cases}\max _{u_{k+1} \in \mathcal{U}_{k+1, x}^{F e e d b a c k}} \mathbb{E}\left(\phi_{k+1}\left(A_{k+1}^{u}\right) \mid A_{k}^{u}=x\right), & \left(c-\sum_{\vartheta=1}^{\vartheta_{\max }} x_{c}(\vartheta)\right) \lambda>k, \\ J(x) & \text { otherwise, }\end{cases}
$$

where the function $J$ is given in (13), $\mathcal{U}_{k+1, x}^{\text {Feedback }}$ is the set of strategies of the form $U_{k+1}(x, y)$ (for $x \in \mathcal{A}, y \in \mathcal{O})$ with values in $\left\{0, \max \left(1, M-i_{c}-i_{p}\right)\right\}$, and with $A_{k+1}^{u}$ given from (15) by

$$
A_{k+1}^{u}=f\left(A_{k}^{u}, Y_{k+1}, U_{k+1}\left(A_{k}^{u}, Y_{k+1}\right)\right) .
$$

The expectation in (16) is understood as the conditional law of $Y_{k+1}$ on the event $\left\{A_{k}^{u}=x\right\}$. We are guaranteed that $\phi_{m}(x)=J(x)$ for all $x \in \mathcal{A}$. We can further write that control only occurs at distance to failure 1 (Proposition 3.4),

$$
\begin{aligned}
\phi_{k}(x)= & \sum_{\vartheta>1} p_{k}(1, \vartheta) \phi_{k+1}\left(x^{\prime}=x_{c}-e_{\vartheta}+e_{\vartheta-1}, x_{p}, i_{c}, i_{p}\right) \\
& +p_{k}(1,1) \max \left\{\phi_{k+1}\left(x^{\prime}=x_{c}-e_{\vartheta}, x_{p}, i_{c}, i_{p}\right), \phi_{k+1}\left(x^{\prime}=x_{c}, x_{p}, i_{c}+1, i_{p}\right) \mathbb{1}_{i_{c}+1, i_{p}<M}\right\} \\
& +p_{k}(0,0) \max \left\{\phi_{k+1}\left(x^{\prime}=x_{c}, x_{p}-1, i_{c}, i_{p}\right), \phi_{k+1}\left(x^{\prime}=x_{c}, x_{p}, i_{c}, i_{p}+1\right) \mathbb{1}_{i_{c}+1, i_{p}<M}\right\} \\
& \quad+\left(1-\sum_{\vartheta \geq 1} p_{k}(1, \vartheta)-p_{k}(0,0)\right) \phi_{k+1}(x),
\end{aligned}
$$

where $e_{\vartheta}$ is the $\vartheta$ th unit vector in $\mathbb{R}^{\theta_{\max }}$ and the transition probabilities are given in (14).

The first line corresponds to the case when a core bank at distance to failure $>1$ is chosen. The second line corresponds to the case when a core bank at distance to failure 1 is chosen. The third line corresponds to the case when a peripheral bank is chosen. The last line corresponds to the case when the chosen bank is a failed core bank.

4.2. Numerical solution and policy implications. We solve Problem 1 by implementing the dynamic programming equation (17) with the numerical values of the parameters given in Table 1, unless otherwise stated.

Connectivity and value of the financial system. We first compare the value of the financial system under different connectivities and different intervention budgets. We understand by the value of the financial system the value of the optimal control problem (1). The efficiency of the network is fixed, and given by $\pi=0.5$, which means that credit from the external creditor to the peripheral banks is intermediated, on average, by two peripheral banks.

Figure 3 plots the value of the financial system, as a function of connectivity and the initial condition of the system, captured by the initial number of nonfailed core banks. When the initial state of the system is such that there are no failed banks $\left(C_{0}\left(\vartheta_{\max }\right)=c\right)$, then the value 
Table 1

Parameters of the stylized model.

\begin{tabular}{|c|c|}
\hline Number of core banks & $c=9$ \\
Network efficiency & $\pi=.5$ \\
Number of projects of core banks & $y_{c}=1$ \\
Number of projects of peripheral banks & $y_{p}=1$ \\
Connectivity & $\lambda \in[0,24]$ \\
Maximum distance to failure & $\vartheta_{\max }=3$ \\
Intervention budget & $M=4$ \\
\hline
\end{tabular}

of the system always increases with connectivity. When there is at least one initially failed bank in the system, $C_{0}\left(\vartheta_{\max }\right)<c$, the relation between value and connectivity is nonmonotonous: it first increases with connectivity and then decreases with connectivity. This holds for the case without intervention and for various intervention budgets. A priori, connectivity leads to the creation of new projects, which increases the value of the financial system. However, when there are initially failed banks in the system, contagion also increases and the value of the system is driven by these two opposite effects. This means that above a certain level of connectivity, which depends on the initial state of the system, the negative effect of contagion becomes predominant and the value of the system decreases with connectivity. Therefore, there is an optimal level of connectivity for each initial state for a given budget, and this optimum is increasing in the budget of the controller. Another important observation is that if connectivity becomes too large, the value of the financial system drops below the value of the disconnected system (connectivity $\lambda=0$ ).

Next, in Figure 4 we look more closely at the difference in value between the controlled system and the uncontrolled system. The results show that intervention is most efficient, i.e., leads to the largest increase in value, when there are few initial failures and the connectivity is large. In this case, intervention can significantly mitigate contagion and the benefits of large connectivity are maintained. As the number of initial failures increases, the connectivity at which intervention is efficient becomes smaller and smaller. Note that the preserved value (difference in value with intervention and without intervention) always surpasses the intervention budget.

We now describe the policy of the controller. We compare the number of interventions on core and peripheral banks for varying connectivity. Figure 5 plots the difference in the expected number of interventions on the core and peripheral banks. We set $y_{c}=10, y_{p}=1$ to ensure that intervention on peripheral banks is not driven by the fact that the size of core banks is too small. When there are no failed core banks initially in the system, we verify that there are also no interventions as there is no contagion in the system.

When there is at least one initially failed core bank in the system, we distinguish the following pattern. In the low connectivity regime, the number of interventions on peripheral banks increases with connectivity. Above a certain level of connectivity (medium connectivity regime) it is optimal to increase the number of interventions on core banks. Then, in the high connectivity regime, it is again optimal to intervene towards the peripheral banks. This can be interpreted as follows. In the low connectivity regime, there is a small number of links among core banks and core banks can absorb first order contagion effects easily (first order contagion 



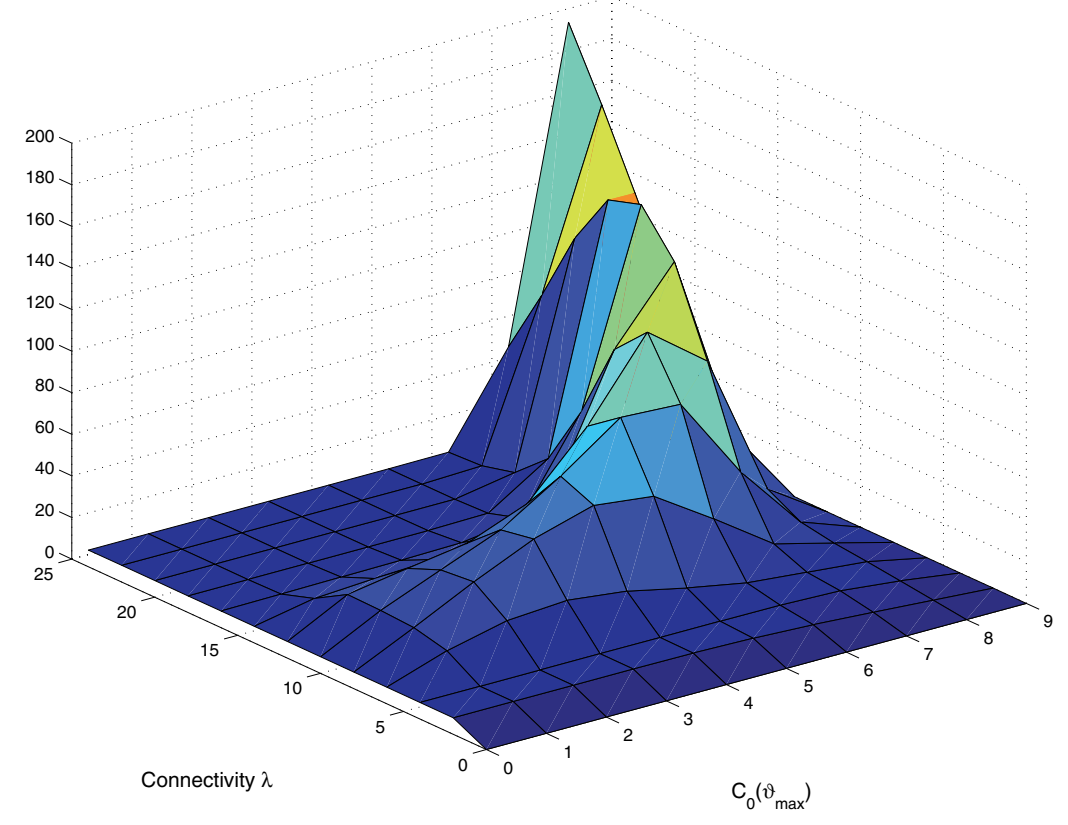

Figure 4. Difference between the values of the system in the case with intervention $(M=4)$ and without intervention.

refers to contagion between a bank and its direct borrowers). In this case the controller intervenes directly on the peripheral banks which borrow from the failed core banks. In the medium connectivity regime, core banks get connected to other core banks and there is higher order contagion among core banks (higher order contagion refers to contagion from a bank to its borrowers and borrowers of borrowers, etc.). Core banks can no longer absorb contagion effects by themselves. It is then optimal to intervene on core banks in order to prevent this type of contagion. Then in the high connectivity regime, the number of links among core banks is so large that contagion among them cannot be stopped under the given budget. It is then again optimal to intervene on the peripheral banks to stop at least the contagion from cores to peripheral. This effect holds even if the number of projects of core banks is much larger than the number of projects of peripheral banks, so it is a connectivity-driven effect.

Efficiency and the value of the financial system. The results so far hold under a fixed efficiency level in the network. We now study the impact of the efficiency level on the intervention policy. We keep the number of credit lines from core to peripheral banks constant and vary the efficiency. Comparing Figure 6 with Figure 5 shows that decreasing efficiency in a network with a fixed number of connections from core to peripheral banks has a similar effect on the intervention policy as increasing the overall connectivity for fixed efficiency level.

Here the relation with the number of intermediaries and potential of higher order contagion among core banks is obvious, since the number of funded projects of peripheral banks does not change as we vary the efficiency. The only thing that changes is the number of in- 


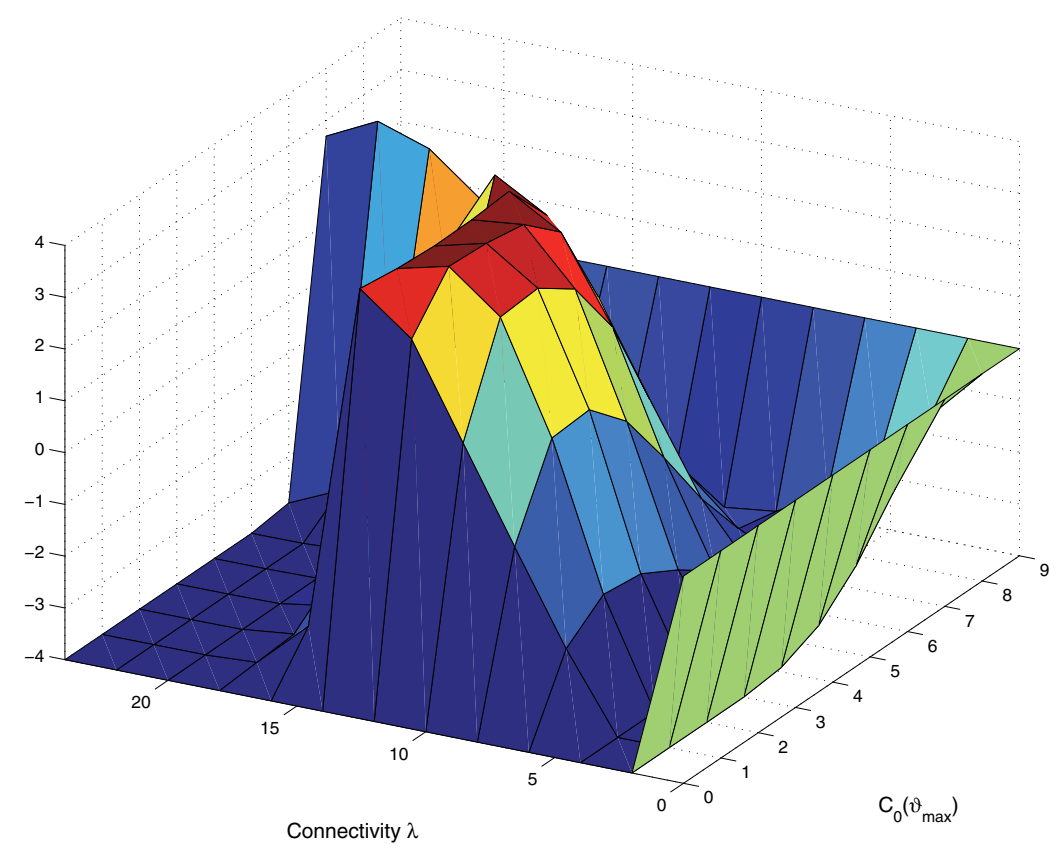

Figure 5. Difference between the expected number of interventions on core and peripheral banks as a function of connectivity, $y_{p}=1, y_{c}=10$.

termediary core banks. For highly efficient systems $(\pi=1)$, credit from the external creditor to the peripheral banks passes only through one core bank. Since there is no potential of contagion among core banks, intervention will always be targeted at peripheral banks. As we decrease efficiency, the number of intermediaries increases, and chains of intermediaries emerge. When the efficiency is sufficiently low, there is higher order contagion among core banks and intervention is targeted on core banks. For highly inefficient systems, higher order contagion among core banks cannot be stopped since there can be cycles among core banks, and again intervention is targeted at peripheral banks.

One of the important implications of our work is to support the periphery rather than the core if the core is overconnected. There results could be extrapolated, and would mean to lend directly to the real economy in the high connectivity regime, conditional on a shock. Our results suggest practical ways to assess whether the core is overconnected in terms of efficiency, where efficiency is defined as the percentage of the credit extended by core banks to the periphery. While the network model is stylized and overconnectivity is not directly quantifiable, efficiency on the other hand is.

5. Conclusions and further research directions. The most important insight of this paper relates the value of the financial system, connectivity, and optimal intervention. Up to a certain connectivity, the value of the financial system increases with connectivity. This implies that a connected system prone to contagion and thus depending on intervention is preferable to 


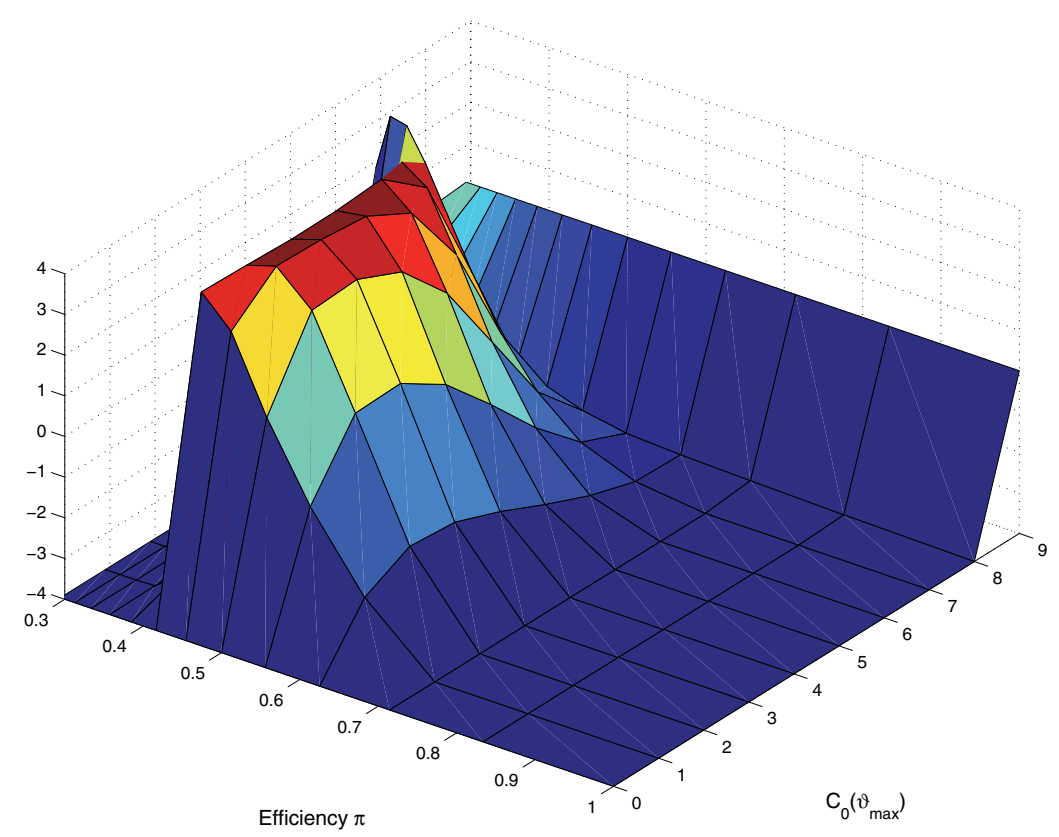

Figure 6. Difference between the expected number of interventions on core and peripheral banks as a function of network efficiency, $y_{p}=1, y_{c}=10$.

a disconnected system. However, this is no longer the case if connectivity becomes too large and even in the case there is intervention, the value of the system may fall below the value of the disconnected system. We identify the optimal policy of a controller that injects liquidity into the system so as to maximize its expected value. We find that in the low connectivity regime the controller prefers to inject directly into the periphery. In the medium connectivity regime, the policy switches and it is preferable to inject liquidity into the core banks.

Somewhat surprisingly, this is no longer true if the connectivity is high: in the highest connectivity regime, it is again optimal to intervene into the periphery rather than the core. This insight shows that it is far from obvious that connectivity of a core bank should always be brought forward as an argument for priority intervention and it may be sometimes preferable to invest in noncore banks that lend directly to the economy. One implication is that setting limits on connectivity is a good policy for limiting systemic risk only in the highest connectivity regime, and otherwise it is preferable to create an intervention fund (possibly using banks' contributions) that can be used to maximize the benefits of connectivity.

We have introduced a stylized model, which despite its simplicity suggests that there is an optimal level of connectivity for the financial system. However, the system is dependent on intervention at that level of connectivity, in case there are initial failures. The natural question remains how to create incentives for the banks to attain an optimal level of connectivity and how to design a guarantee fund of the banks that would represent the intervention fund. Results similar to those in this paper could be obtained for a tiered structured with more 
than two levels of banks. Given that optimal control will happen at certain optimal levels, this will drive the banks' incentives to join a certain level in the tiered financial structure.

There are many ways in which more realism can be added to the model. Of course, the mechanical behavior of banks that withdraw all credit lines and fully liquidate their portfolios as soon as they reach a distance to failure zero is highly stylized as banks are strategic about which lines to withdraw and how much to liquidate. Relaxing the assumption of full liquidation and full withdrawal of credit lines in case of failures is left for future research.

Other extensions include the design of optimal sharing rules of the benefit from connectivity and control among the controller and the financial system, for example in the form of interest. In this model, we do not consider the possibility that the distance to failure of a bank can exogenously rise. Therefore, the model is more adapted to a short term crisis. It would be an interesting extension to allow for an exogenous rise in the distance to failure.

Acknowledgments. We are indebted to Jean-Philippe Chancelier for valuable discussions during several meetings of our group on Systemic Risk at INRIA as well as for his help with the setup of the model. We thank the anonymous referees for their remarks that helped to improve this paper. We thank the participants of the PIMS Workshop on Systemic Risk and Financial Networks, 2014, the Newton Institute Program on Systemic Risk, 2014, the Bachelier Seminar, Paris, 2014, and the Workshop on Network Formation and Systemic Risk at The University of Pennsylvania Warren Center, 2015, and the Cornell Research Workshop on Data Analytics at Johnson Graduate School of Management, 2015, for helpful comments.

\section{REFERENCES}

[1] D. Acemoglu, A. Ozdaglar, and A. Tahbaz-Salehi, Systemic risk and stability in financial networks, Am. Econ. Rev., 105 (2015), pp. 564-608.

[2] V. V. Acharya And D. SkeIe, A model of liquidity hoarding and term premia in inter-bank markets, J. Monetary Econ., 58 (2011), pp. 436-447.

[3] V. V. Acharya, D. Gale, And T. Yorulmazer, Rollover risk and market freezes, J. Finance, 66 (2011), pp. 1177-1209.

[4] H. Amini, R. Cont, And A. MincA, Resilience to contagion in financial networks, Mathematical Finance, to appear.

[5] H. Amini And A. Minca, Mathematical modeling of systemic risk, in Advances in Network Analysis and Its Applications, Mathematics in Industry 18, Evangelos Kranakis, ed., Springer, Berlin, Heidelberg, 2013, pp. 3-26.

[6] S. Battiston, D. Delli Gatti, M. Gallegati, B. Greenwald, and J. E. Stiglitz, Liaisons dangereuses: Increasing connectivity, risk sharing, and systemic risk, J. Econ. Dyn. Control, 36 (2012), pp. 1121-1141.

[7] D. P. Bertsekas and S. E. Shreve, Stochastic Optimal Control: The Discrete Time Case, Mathematics in Science and Engineering 139, Academic Press, New York, 1978.

[8] L. Blume, D. Easley, J. Kleinberg, R. Kleinberg, and E. Tardos, Network formation in the presence of contagious risk, ACM Transactions on Economics and Computation, 1 (2013), 6.

[9] M. Boss, H. Elsinger, M. Summer, And S. Thurner, Network topology of the interbank market, Quantitative Finance, 4 (2004), pp. 677-684.

[10] R. Carmona, J.-P. Fouque, And L.-H. Sun, Mean field games and systemic risk, Commun. Math. Sci., 13 (2015), pp. 911-933.

[11] R. Cont, A. Moussa, And E. B. SAntos, Network structure and systemic risk in banking systems, in Handbook of Systemic Risk, J.-P. Fouque and J. Langsam, eds., Cambridge University Press, Cambridge, UK, 2012, pp. 327-368. 
[12] B. Craig and G. Von Peter, Interbank tiering and money center banks, J. Financ. Intermed., 23 (2014), pp. 322-347.

[13] D. W. Diamond and P. H. Dybvig, Bank runs, deposit insurance, and liquidity, J. Polit. Econ., 91 (1983), pp. 401-419.

[14] D. Duffie, N. GÂrleanu, and L. H. Pedersen, Over-the-counter markets, Econometrica, 73 (2005), pp. $1815-1847$.

[15] L. Eisenberg And T. H. Noe, Systemic risk in financial systems, Manag. Sci., 47 (2001), pp. 236-249.

[16] S. ERoL, Network hazard and bailouts, working paper, 2015

[17] H. Föllmer, Spatial risk measures and their local specification: The locally law-invariant case, Stat. Risk Model., 31 (2014), pp. 79-101.

[18] J.-P. Fouque And T. ICHIBA, Stability in a model of interbank lending, SIAM J. Financial Math., 4 (2013), pp. 784-803.

[19] J.-P. Fouque and J. A. Langsam, Handbook on Systemic Risk, Cambridge University Press, Cambridge, UK, 2013.

[20] V. Fourel, J.-C. HÉam, D. Salakhova, and S. Tavolaro, Domino Effects when Banks Hoard Liquidity: The French Network, Tech. report, Banque de France, Paris, France, 2013.

[21] P. Gai, A. Haldane, And S. Kapadia, Complexity, concentration and contagion, J. Monetary Econ., 58 (2011), pp. 453-470.

[22] P. Gai And S. Kapadia, Liquidity hoarding, network externalities, and interbank market collapse, Proc. R. Soc. Lond. Ser. A Math. Phys. Eng. Sci., 466 (2010), pp. 2401-2423.

[23] J. Garnier, G. Papanicolaou, and T.-W. Yang, Diversification in financial networks may increase systemic risk, in Handbook on Systemic Risk, Cambridge University Press, Cambridge, 2013, pp. 432443.

[24] K. Giesecke And B. Kim, Systemic risk: What defaults are telling us, Manag. Sci., 57 (2011), pp. 13871405.

[25] Z. He And W. Xiong, Dynamic debt runs, Rev. Financ. Stud., 25 (2012), pp. 1799-1843.

[26] A. Krishenik, A. MincA, AND J. Wissel, When do creditors with heterogeneous beliefs agree to run?, Finance Stoch., 19 (2015), pp. 233-259.

[27] A. Minca AND A. Sulem, Optimal control of interbank contagion under complete information, Stat. Risk Model., 31 (2014), pp. 23-48.

[28] A. Minca And J. Wissel, Dynamics and stability of debt capacity, available at SSRN 2619498, 2015.

[29] S. MorRis AND H. S. SHIn, Illiquidity component of credit risk, working paper, 2009.

[30] J. MüLLER, Interbank credit lines as a channel of contagion, J. Financ. Serv. Res., 29 (2006), pp. 37-60.

[31] L. C. G. Rogers And L. AM VeraArt, Failure and rescue in an interbank network, Manag. Sci., 59 (2013), pp. 882-898.

[32] R. VAn Der Hofstad, Random graphs and complex networks, available online at http://www.win.tue. $\mathrm{nl} / \sim$ rhofstad/NotesRGCN.html, 2009.

[33] D. J. WATts, A simple model of global cascades on random networks, Proc. Natl. Acad. Sci. USA, 99 (2002), pp. 5766-5771. 